\title{
Toward a Combinatorial Proof of the Jacobian Conjecture!
}

\author{
Dan Singer \\ Department of Mathematics and Statistics \\ Minnesota State University, Mankato, U.S.A. \\ dan. singer@mnsu .edu
}

Submitted: Apr 30, 2011; Accepted: Nov 18, 2011; Published: Nov 28, 2011

Mathematics Subject Classifications: 05C99, 05E99, 14R15, 15A03

\begin{abstract}
The Jacobian conjecture [Keller, Monatsh. Math. Phys., 1939] gives rise to a problem in combinatorial linear algebra: Is the vector space generated by rooted trees spanned by forest shuffle vectors? In order to make headway on this problem we must study the algebraic and combinatorial properties of rooted trees. We prove three theorems about the vector space generated by binary rooted trees: Shuffle vectors of fixed length forests are linearly independent, shuffle vectors of nondegenerate forests relative to a fixed tree are linearly independent, and shuffle vectors of sufficient length forests are linearly independent. These results are proved using the acyclic digraph method for establishing that a coefficient matrix has full rank [Singer, The Electronic Journal of Combinatorics, 2009]. We also provide an infinite class of counterexamples to demonstrate the need for sufficient length in the third theorem.
\end{abstract}

\section{Introduction}

Every since reading Doron Zeilberger's paper "Toward a Combinatorial Proof of the Jacobian Conjecture?" as a graduate student in the nineties [9], I have been thinking about this question. Zeilberger proposed that the Joyal method of combinatorial species be brought to bear on the problem [2]. Wright has formulated a combinatorial approach to the Jacobian conjecture using trees [7], and I have formulated a slightly different version using rooted trees [4]. See also [1] and [8]. What makes the combinatorial approach so difficult is that one is attempting to prove a much stronger statement than the Jacobian conjecture itself: individual weights of trees, which contribute terms to the inverse of a polynomial system, are equal to zero. We don't even know that the Jacobian conjecture is true, let alone the truth of the combinatorial statements that would imply it. What is 
required here is a systematic study of the relevant combinatorial and algebraic properties of trees.

In this paper I will describe the progress I have made in this regard, in particular Theorems 5.3, 6.3, 7.15, and Example 8.1. The content of the theorems is that, in the vector space generated by rooted binary trees, shuffle vectors of fixed length forests are linearly independent; shuffle vectors of nondegenerate forests arising from a single tree are linearly independent; and shuffle vectors of all sufficient length forests are linearly independent. Example 8.1 provides an infinite number of counterexamples that demonstrate the need for sufficient length. My toolkit includes the acyclic digraph method for proving that a coefficient matrix has full rank [5].

To simplify the presentation we will deal with the quadratic case of the Jacobian conjecture only, which has the advantage of already having been proved true using algebraic methods [6]. The general idea is that the formal inverse of a system of polynomials can be expressed as an infinite sum of weights of rooted binary trees. We wish to prove that all but a finite number of these weights are equal to zero. The jacobian condition implies that certain linear combinations of tree weights are equal to zero, and the combinatorial task is to show that all but a finite number of tree weights can be spanned by the zero linear combinations. To this end we lift the problem statement from polynomials to trees. We will spend a few paragraphs here to introduce some terminology and make our statements more precise.

\section{Rooted Binary Trees}

Rooted binary trees are isomorphism classes of rooted trees in which each parent vertex has two children. Each rooted binary tree with more than one leaf can be uniquely represented in the form of a two-element multiset: $T=\{A, B\}$, where $A$ and $B$ are the rooted binary trees whose roots are the children of the root of $T$. Since rooted binary trees is a mouthful, from now on we will just say trees. We order trees recursively as follows: the smallest tree is $T_{1}$, the tree consisting of one leaf vertex and no edges. More generally, if $S$ and $T$ are trees, $S=\{A, B\}, T=\left\{A^{\prime}, B^{\prime}\right\}, A \geq B$, and $A^{\prime} \geq B^{\prime}$, then we say that $S>T$ if and only if $S$ has more leaves than $T$ or they have the same number of leaves and $(A, B)>\left(A^{\prime}, B^{\prime}\right)$ in lexicographic order.

Given this ordering we label the trees $T_{1}, T_{2}, \ldots$ in increasing order. The totally symmetric trees in this list are

$$
S_{0}=T_{1}, S_{1}=\left\{S_{0}, S_{0}\right\}, \ldots, S_{k}=\left\{S_{k-1}, S_{k-1}\right\} .
$$

We have

$$
S_{0}=T_{1}, S_{1}=T_{2}, S_{2}=T_{4}, S_{3}=T_{26},
$$

and so on. We associate with each tree $T_{i}$ a non-commuting variable $t_{i}$, and we associate with each word $t_{i_{1}} \ldots t_{i_{k}}$ the expresson $\operatorname{tree}\left(t_{i_{1}} \ldots t_{i_{k}}\right)$ defined recursively by

$$
\operatorname{tree}\left(t_{i}\right)=\left\{T_{1}, T_{i}\right\}
$$


and

$$
\operatorname{tree}\left(t_{i_{1}} t_{i_{2}} \ldots t_{i_{k}}\right)=\left\{\operatorname{tree}\left(t_{i_{1}} t_{i_{2}} \ldots t_{i_{k-1}}\right), T_{i_{k}}\right\}
$$

For example we have

$$
\operatorname{tree}\left(t_{1}\right)=S_{1}, \operatorname{tree}\left(t_{1} t_{2}\right)=S_{2}, \text { tree }\left(t_{1} t_{2} t_{4}\right)=S_{3} .
$$

The first eight trees are depicted in Figure 2.1. The root vertices are filled.

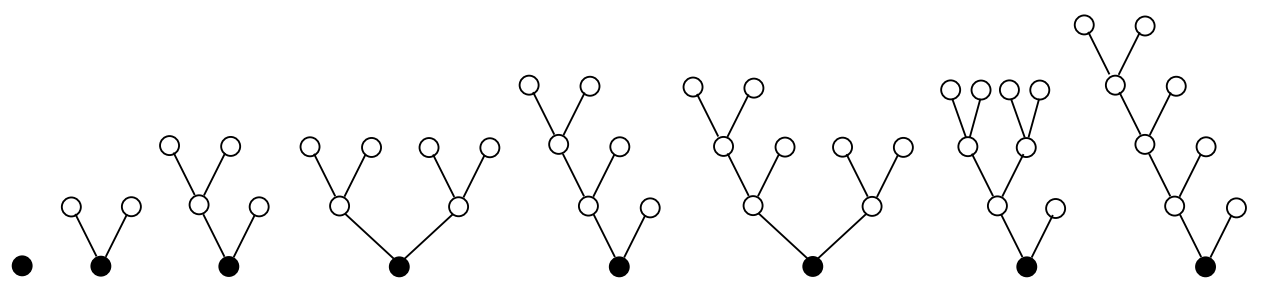

Figure 2.1: $T_{1}, T_{2}, T_{3}, T_{4}, T_{5}, T_{6}, T_{7}, T_{8}$

Given a rational finite linear combination

$$
\sum_{i_{1}, \ldots, i_{k}} q_{i_{1}, \ldots, i_{k}} t_{i_{1}} \cdots t_{i_{k}}
$$

we define

$$
\operatorname{tree}\left(\sum_{i_{1}, \ldots, i_{k}} q_{i_{1}, \ldots, i_{k}} t_{i_{1}} \cdots t_{i_{k}}\right)=\sum_{i_{1}, \ldots, i_{k}} q_{i_{1}, \ldots, i_{k}} \operatorname{tree}\left(t_{i_{1}} \cdots t_{i_{k}}\right)
$$

implicitly defining a rational vector space $P_{\mathcal{B}}$ consisting of polynomials in the noncommuting variables $t_{1}, t_{2}, \ldots$, and another rational vector space $V_{\mathcal{B}}$ with basis $\mathcal{B}=\left\{T_{1}, T_{2}, \ldots\right\}$. To compete the description of tree as a linear operator on $P_{\mathcal{B}}$ we define

$$
\operatorname{tree}(q)=q T_{1}
$$

for all $q \in \mathbb{Q}$ and extend by linearity to all polynomials. The tree operator is not injective: for example, we have tree $\left(t_{1} t_{1}\right)=\operatorname{tree}\left(t_{2}\right)=t_{3}$.

Let $t_{e_{1}} \cdots t_{e_{k}}$ be a word in the variables $t_{1}, t_{2}, \ldots$ The shuffle polynomial corresponding to this word is the symmetric function

$$
\left\langle t_{e_{1}} \cdots t_{e_{k}}\right\rangle=\sum_{\sigma \in \mathcal{S}_{k}} t_{e_{\sigma(1)}} \cdots t_{e_{\sigma(k)}}
$$

The shuffle vector in $V_{\mathcal{B}}$ corresponding to the word $t_{e_{1}} \cdots t_{e_{k}}$ is

$$
S\left(t_{e_{1}} \cdots t_{e_{k}}\right)=\operatorname{tree}\left(\left\langle t_{e_{1}} \cdots t_{e_{k}}\right\rangle\right) \text {. }
$$

For example, we have

$$
\begin{gathered}
S\left(t_{1} t_{1} t_{2}\right)=\operatorname{tree}\left(\left\langle t_{1} t_{1} t_{2}\right\rangle\right)=\operatorname{tree}\left(2 t_{1} t_{1} t_{2}+2 t_{1} t_{2} t_{1}+2 t_{2} t_{1} t_{1}\right)= \\
2 T_{6}+2 T_{7}+2 T_{8} .
\end{gathered}
$$




\section{Jacobian Conjecture}

In [4] I show that given a system of polynomials

$$
\left(F_{1}, \ldots, F_{n}\right)=\left(x_{1}-H_{1}, \ldots, x_{n}-H_{n}\right) \in \mathbb{C}\left[x_{1}, \ldots, x_{n}\right]^{n},
$$

each polynomial $H_{i}$ homogeneous of total degree 2 , there is a linear mapping

$$
\phi: V_{\mathcal{B}} \rightarrow \mathbb{C}\left[x_{1}, \ldots, x_{n}\right]^{n}
$$

with the property that if we set

$$
\left(G_{1}, \ldots, G_{n}\right)=\sum_{T \in \mathcal{B}} \phi(T)
$$

then we have

$$
G_{i}-H_{i}\left(G_{1}, \ldots, G_{n}\right)=x_{i}
$$

for $1 \leq i \leq n$, where $\mathcal{B}$ is the set of rooted binary trees. The expressions $G_{1}, \ldots, G_{n}$ are formal power series in the variables $x_{1}, \ldots, x_{n}$, and if $T$ is a tree with $v$ leaves then $\phi(T)$ is a vector of polynomials each homogeneous of total degree $v$. The quadratic case of the Jacobian conjecture states that if the determinant of the jacobian of the polynomial system $\left(F_{1}, \ldots, F_{n}\right)$ is equal to a non-zero complex number then each $G_{i}$ is a polynomial of finite degree. The combinatorial problem is to show that $\phi(T)=0$ for all trees $T$ with sufficiently many leaves. The fact that the jacobian determinant is a non-zero constant and the polynomial system $\left(H_{1}, \ldots, H_{n}\right)$ is homogeneous implies that the jacobian matrix of the latter system is nilpotent of index $n$.

This has the following combinatorial significance: assuming $\left(\frac{\partial H_{i}}{\partial x_{j}}\right)^{\delta}=0$, for each triple of words $\left(x_{1} \cdots x_{a}, y_{1} \cdots y_{\delta}, z_{1} \cdots z_{b}\right)$ we have

$$
\phi\left(\operatorname{tree}\left(x_{1} \cdots x_{a} \cdot\left\langle y_{1}, \ldots y_{\delta}\right\rangle \cdot z_{1} \cdots z_{b}\right)\right)=0 .
$$

So one way to supply a combinatorial proof of the quadratic case of the Jacobian conjecture is to show that every binary tree with a sufficient number of leaves can be expressed as a linear combination of shuffle vectors of the form

$$
\operatorname{tree}\left(x_{1} \cdots x_{a} \cdot\left\langle y_{1} \cdots y_{\delta}\right\rangle \cdot z_{1} \cdots z_{b}\right)
$$

I was able to do this for all rooted binary trees with at least seven leaves under the assumption that $\delta=3$ ([4], Theorem 5.3). The ordering of trees I have described above played a key role in the proof. 


\section{The Acyclic Digraph Method}

In the next three sections we will present three theorems which describe conditions under which the shuffle vectors $S\left(t_{e_{1}} \cdots t_{e_{k}}\right)$ defined in Equation 2.1 above are linearly independent in $V_{\mathcal{B}}$. Each theorem sheds a little light on algebraic and combinatorial properties of trees that may one day prove useful for proving the Jacobian conjecture (the quadratic case, at any rate). The theorems are presented in the order corresponding to the length of time it took me to prove them (ten minutes, one week, two years) and, I think, in order of increasing importance. We will use the acyclic digraph method to prove each theorem [5], restating Definition 2.1, Definition 2.3, and Theorem 2.11 from that paper for easy reference:

Definition 4.1. Let $A=\left(a_{i j}\right)$ be a real $m \times n$ matrix. The matrix $A$ gives rise to an edge-labeled digraph $G_{A}=\left(V_{A}, E_{A}\right)$, with vertex set $V_{A}=\left\{v_{1}, \ldots, v_{n}\right\}$ and for all $(j, i, k) \in[n] \times[m] \times[n]$ a directed edge $\left(v_{j}, i, v_{k}\right)$ from $v_{j}$ to $v_{k}$ labeled $i$ if and only if $a_{i j} a_{i k} \neq 0$.

Definition 4.2. Let $A=\left(a_{i j}\right)$ be a real $m \times n$ matrix with no zero columns, and let $G_{A}$ be the associated edge-labeled digraph as in Definition 4.1. For each column $j \leq n$ we define $R_{j}=\left\{i \leq m: a_{i j} \neq 0\right\}$. Since $A$ has no zero columns, every set $R_{j}$ is non-empty. Given a row selection function $r: V_{A} \rightarrow\{1, \ldots, m\}$ which satisfies $r\left(v_{j}\right) \in R_{j}$ for all $j \leq n$ we form the row selection subgraph $G_{r}=\left(V_{A}, E_{r}\right)$ of $G_{A}$ with vertex set $V_{A}$ and edge set

$$
E_{r}=\left\{\left(v, i, v^{\prime}\right) \in E_{A}: i=r(v)\right\} .
$$

The acyclic digraph method provides a criterion whereby a coefficient matrix has full rank:

Theorem 4.3. Let $A=\left(a_{i j}\right)$ be a $m \times n$ matrix over the reals with no zero columns, let $G_{A}$ be the associated edge-labeled directed graph described in Definition 4.1, let

$$
r: V_{A} \rightarrow\{1, \ldots, m\}
$$

be a row-selection function which satisfies $r\left(v_{j}\right) \in R_{j}$ for all $j \leq n$, and let $G_{r}$ be the row selection subgraph of $G_{A}$ defined by $r$ described in Definition 4.2. If $G_{r}$ has no directed cycles of length $\geq 2$ then the rows chosen by the row-selection function $r$ are linearly independent.

\section{$5 \quad$ Shuffles of Fixed Length Forests}

The main result of this section is Theorem 5.3. Preliminaries: 
Definition 5.1. Let $t_{1}, t_{2}, \ldots$ be the sequence of non-commuting variables referenced in Section 2. We order the variables by $t_{1}<t_{2}<\ldots$ and order words (products of variables) according to lexicographic order of variables. The largest rearrangement of a word $w$ is $\bar{w}$. If $w=\bar{w}$ then we say that $w$ is ordered. We also say that $1<w$ for all words $w$.

Lemma 5.2. Let $x$ and $y$ be words in $t_{1}, t_{2}, \ldots$ of length $k \geq 1$, and assume that $x$ is ordered. If $\operatorname{tree}(x)=\operatorname{tree}(y)$ then $x \leq \bar{y}$.

Proof. By induction on $k$. When $k=1$ we have $x=y$. Assume the property is true for words of length $k$. Consider $x$ and $y$ of length $k+1$. Write $x=t_{e_{1}} t_{e_{2}} \cdots t_{e_{k+1}}$ and $y=t_{f_{1}} t_{f_{2}} \cdots t_{f_{k+1}}$ with $\operatorname{tree}(x)=\operatorname{tree}(y)$ and $e_{1} \geq e_{2} \geq \cdots \geq e_{k+1}$. Then we have equality of the multisets $\left\{\operatorname{tree}\left(t_{e_{1}} \cdots t_{e_{k}}\right), T_{e_{k+1}}\right\}$ and $\left\{\operatorname{tree}\left(t_{f_{1}} \cdots t_{f_{k}}\right), T_{f_{k+1}}\right\}$. If $e_{k+1}=f_{k+1}$ then

$$
\operatorname{tree}\left(t_{e_{1}} \cdots t_{e_{k}}\right)=\operatorname{tree}\left(t_{f_{1}} \cdots t_{f_{k}}\right) \text {, }
$$

hence by the induction hypothesis $t_{e_{1}} \cdots t_{e_{k}} \leq \overline{t_{f_{1}} \cdots t_{f_{k}}}$, hence

$$
x=t_{e_{1}} t_{e_{2}} \cdots t_{e_{k+1}} \leq \overline{t_{f_{1}} \cdots t_{f_{k}}} t_{f_{k+1}} \leq \bar{y}
$$

But if $e_{k+1} \neq f_{k+1}$ then

$$
T_{f_{k+1}}=\operatorname{tree}\left(t_{e_{1}} \cdots t_{e_{k}}\right)>T_{e_{1}}
$$

by virtue of the fact that $T_{f_{k+1}}$ has more vertices than $T_{e_{1}}$, hence $f_{k+1}>e_{1}$, hence $x<\bar{y}$.

Theorem 5.3. For each $k \geq 1$ the shuffle vectors in the set

$$
\{S(w): w \text { is an ordered word of length } k\}
$$

are linearly independent in $V_{\mathcal{B}}$.

Proof. Let $k \geq 1$ be given. It will suffice to restrict ourselves to ordered words $w$ such that $\operatorname{tree}(w)$ has $v$ leaves. Having fixed $k$ and $v$, let $X_{1}<X_{2}<\cdots<X_{p}$ be the list of trees with $v$ leaves and let $w_{1}<w_{2}<\cdots<w_{q}$ be the list of ordered words yielding trees with $v$ leaves. Let $A=\left(a_{i j}\right)$ be $p \times q$ matrix in which each column $j$ contains the coefficients of $S\left(w_{j}\right)$ expanded in terms of the ordered basis $X_{1}, \ldots, X_{p}$. Construct $G_{A}$ as in Definition 4.1. We define our row selection function

$$
r:\left\{w_{1}, \ldots, w_{q}\right\} \rightarrow\left\{X_{1}, \ldots, X_{p}\right\}
$$

via

$$
r\left(w_{j}\right)=\operatorname{tree}\left(w_{j}\right)
$$


naming the vertices $w_{1}, \ldots, w_{q}$ instead of $v_{1}, \ldots, v_{q}$ and naming the edge labels $X_{1}, \ldots, X_{p}$ instead of $1, \ldots, p$. We will show that $G_{r}$ has no non-trivial cycles, hence the coefficient matrix has rank $q$ and the shuffle vectors are linearly independent.

The non-loop edges in $G_{r}$ are of the form $\left(w_{i}, \operatorname{tree}\left(w_{i}\right), w_{j}\right)$ where $w_{i}$ and $w_{j}$ are distinct ordered words and tree $\left(w_{i}\right)$ appears in the support of $S\left(w_{j}\right)$. So we have

$$
\operatorname{tree}\left(w_{i}\right)=\operatorname{tree}\left(\sigma\left(w_{j}\right)\right)
$$

for some rearrangement $\sigma\left(w_{j}\right)$ of $w_{j}$. By Lemma 5.2 we have $w_{i} \leq w_{j}$. Since the words are distinct, we have $w_{i}<w_{j}$. Hence the vertices encountered along walks in $G_{r}$ using non-loop edges strictly increase, which implies $G_{r}$ has no non-trivial cycles.

\section{Nondegenerate Shuffles of a Tree}

The main result of this section is Theorem 6.3. Preliminaries:

Definition 6.1. Let $w=t_{e_{1}} \cdots t_{e_{k}}$ be a word in the variables $t_{1}, t_{2}, \ldots$ If $k \geq 1$ then we write $F(w)=T_{e_{1}}+\cdots+T_{e_{k}}$ and interpret this as a multiset of trees rather than as a vector in $V_{\mathcal{B}}$. We refer to $F(w)$ as a forest of tree $(w)$. If $w=1$ then we set $F(w)=0$ and interpret this as the empty multiset. If $F$ and $G$ are forests of trees then we interpret $F+G$ as multiset union (addition of multiplicities) and $F \subseteq G$ as multiset inclusion (every tree in $F$ occurs in $G$ with equal or greater multiplicity). We order forests by $F(u)<F(v)$ if and only if $\bar{u}<\bar{v}$. A forest $F$ is degenerate if $F=k T$ for some positive integer $k$ and tree $T$.

Every forest of a tree $T$ arises in the following way: Let $v$ be an arbitrary leaf vertex in $T$. Then there is a unique path in $T$ from $v$ to the root vertex. Let $v_{0}, v_{1}, \ldots, v_{r}$ be the sequence of vertices encountered along this path in order of occurrence, where $v_{0}=v$ and $v_{r}$ is the root vertex. Let $w_{i}$ be the sibling of $v_{i}$ for $0 \leq i \leq r-1$ and let $T_{e_{i}}$ be the subtree of $T$ rooted at $w_{i}$. Then $T=\operatorname{tree}\left(t_{e_{0}} \cdots t_{e_{r-1}}\right)$ and $T_{e_{0}}+\cdots+T_{e_{r-1}}$ is a forest of $T$.

Proposition 6.2. Let $T$ be a tree and let $F$ be a non-degenerate forest of $T$. Then there exists a tree $\phi(T, F) \neq T$ such that $F$ is a forest of $\phi(T, F)$ and no larger forest of $T$ is a forest of $\phi(T, F)$.

Proof. In summary, the construction is this: let $T=\operatorname{tree}\left(t_{a}^{k} t_{b} w\right)$ and $F=F\left(t_{a}^{k} t_{b} w\right)$ where $a \neq b$. Then

$$
\phi(T, F)= \begin{cases}\operatorname{tree}\left(t_{b} t_{1} w\right) & a=1, k=1 \\ \operatorname{tree}\left(t_{1} t_{b} t_{1}^{k-1} w\right) & a=1, k \geq 2 \\ \operatorname{tree}\left(t_{b} t_{a}^{k} w\right) & a \geq 2 .\end{cases}
$$


We will prove the theorem by induction on $i$ where $T=T_{i}$ among the set of rooted binary trees. The smallest tree with a non-degenerate forest is $T_{4}=\operatorname{tree}\left(t_{1} t_{2}\right)$ and there is only one forest of $T_{4}$, namely $T_{1}+T_{2}$. We set $\phi\left(T_{4}, T_{1}+T_{2}\right)=\operatorname{tree}\left(t_{2} t_{1}\right)=T_{5}$. Now assume the statement of the Proposition is true of all trees $T^{\prime}$ satisfying $T_{4} \leq T^{\prime}<T$ and let $F$ be a non-degenerate forest of $T$. Write $T=\{A, B\}$ and assume without loss of generality

$$
F=F_{A}+B
$$

where $F_{A}$ is a forest of $A$. We will organize the argument by the following cases: $F_{A}=T_{1}$; $F_{A}=k T_{1}$ for some $k \geq 2 ; F_{A}=k C$ for some $k \geq 1$ and $C>T_{1} ; F_{A}$ is non-degenerate. We will write $A=T_{a}$ and $B=T_{b}$.

Case 1: $F_{A}=T_{1}$. Since $F$ is non-degenerate, $B \neq T_{1}$. Since $T=$ tree $\left(t_{1} t_{b}\right)=\left\{T_{2}, B\right\}$, the forests in $T$ are $T_{1}+B=F$ and $F_{B}+T_{2}$ where $F_{B}$ is a forest of $B$. Since $B \geq T_{2}$ and $B$ is larger than any tree in $F_{B}, F$ is the largest forest in $T$. Hence $\phi(T, F)=\operatorname{tree}\left(t_{b} t_{1}\right)=$ $\left\{\operatorname{tree}\left(t_{b}\right), T_{1}\right\} \neq T$ has the required properties. See Figure 6.1.

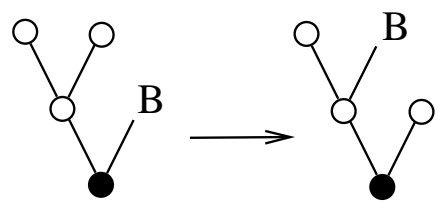

Figure 6.1: Case 1 Shuffle

Case 2: $F_{A}=k T_{1}$ for some $k \geq 2$. Since $F$ is non-degenerate, $B \neq T_{1}$. Since $T=$ tree $\left(t_{1}^{k} t_{b}\right)$, the forests of $T$ are of two types: those containing $B$ and those containing tree $\left(t_{1}^{k}\right)$. Set $\phi(T, F)=\operatorname{tree}\left(t_{1} t_{b} t_{1}^{k-1}\right) \neq T$. The only forest of $\phi(T, F)$ that contains $B$ is $F$. The only forest of $\phi(T, F)$ that could conceivably contain tree $\left(t_{1}^{k}\right)$ is $F_{B}+(k-1) T_{1}+T_{2}$ for some forest $F_{B}$ of $B$, and this forest is no greater than $F=B+k T_{1}$. Hence $\phi(T, F)$ has the required properties. See Figure 6.2.

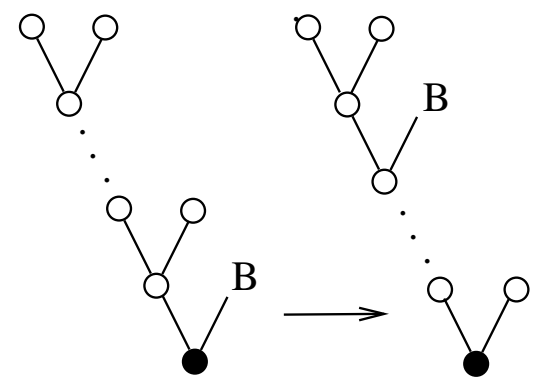

Figure 6.2: Case 2 Shuffle

Case 3: $F_{A}=k C$ for some $k \geq 1$ and $C>T_{1}$. This forces $B \neq C$. Write $C=T_{c}$. Then we have $T=\operatorname{tree}\left(t_{c}^{k} t_{b}\right)$. There are two types of forest in $T$ other than $F=k C+B$ : those 
that contain $\operatorname{tree}\left(t_{c}^{i}\right)$ for some $i \geq 1$, and $F_{C}+(k-1) C+B$ for some forest $F_{C}$ of $C$. The latter is smaller than $F$. Let us set $\phi(T, F)=\operatorname{tree}\left(t_{b} t_{c}^{k}\right)$. The only forest in $\phi(T, F)$ other than $F$ that could conceivable contain tree $\left(t_{c}^{i}\right)$ for some $i \geq 1$ is $F_{B}+T_{1}+k C$ for some forest $F_{B}$ of $B$, and this forest is no greater than $F$. Hence $\phi(T, F)$ has the required properties. Figure 6.3 illustrates this case when $k \geq 2$.

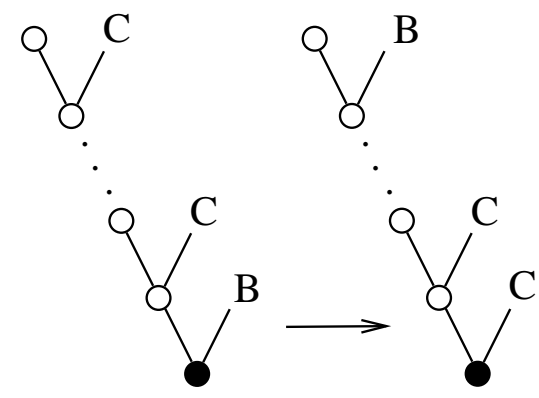

Figure 6.3: Case 3 Shuffle

Case 4: $F_{A}$ is non-degenerate. We will use the induction hypothesis to create a tree $A^{\prime} \neq A$ such that $F_{A}$ is the largest forest of $A$ that belongs to $A^{\prime}$, then set $\phi(T, F)=\left\{A^{\prime}, B\right\}$. See Figure 6.4. Let $G>F$ be a second forest in $T$. We will argue by contradiction that $G$ cannot be a forest of $\phi(T, F)$. Supposing $G$ is also a forest of $\phi(T, F)$, there are four subcases to run down:

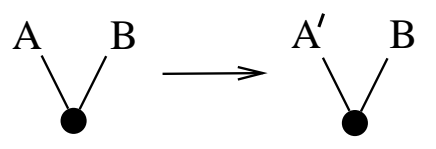

Figure 6.4: Case 4 Shuffle

Case 4.1: $G=F_{A}^{\prime}+B$ for some forest $F_{A}^{\prime}$ of $A$ and $G=F_{A^{\prime}}^{\prime \prime}+B$ for some forest $F_{A^{\prime}}^{\prime \prime}$ of $A^{\prime}$. This implies both that $F_{A}^{\prime}>F_{A}$ and that $F_{A}^{\prime}$ is a forest of $A^{\prime}$, contrary to hypothesis. So this case cannot occur.

Case 4.2: $G=F_{A}^{\prime}+B$ for some forest $F_{A}^{\prime}$ of $A$ and $G=F_{B}^{\prime \prime}+A^{\prime}$ for some forest $F_{B}^{\prime \prime}$ of $B$. This yields

$$
F_{A}^{\prime}+B=F_{B}^{\prime \prime}+A^{\prime}
$$

Since $B \notin F_{B}^{\prime \prime}$, we must have $B=A^{\prime}$ and $F_{A}^{\prime}=F_{B}^{\prime \prime}$. Hence $F_{A}^{\prime}>F_{A}$ and $F_{A}^{\prime}$ is a forest in $A^{\prime}$, contrary to hypothesis. So Case 4.2 is ruled out.

Case 4.3: $G=F_{B}^{\prime}+A$ for some forest $F_{B}^{\prime}$ of $B$ and $G=F_{A^{\prime}}^{\prime \prime}+B$ for some forest $F_{A^{\prime}}^{\prime \prime}$ of $A^{\prime}$. This yields

$$
F_{B}^{\prime}+A=F_{A^{\prime}}^{\prime \prime}+B
$$


Since $A \notin F_{A^{\prime}}^{\prime \prime}$ we must have $B=A$. Given that $F=F_{A}+B$ and that $G>F$, this yields $F_{B}^{\prime}>F_{A}$. But we also have $F_{B}^{\prime}=F_{A^{\prime}}^{\prime \prime}$, making $F_{B}^{\prime}$ a forest in both $A$ and $A^{\prime}$ which is larger than $F_{A}$. Since this is contrary to hypothesis, Case 4.3 cannot occur.

Case 4.4: $G=F_{B}^{\prime}+A$ for some forest $F_{B}^{\prime}$ of $B$ and $G=F_{B}^{\prime \prime}+A^{\prime}$ for some forest $F_{B}^{\prime \prime}$ of $B$. This yields

$$
F_{B}^{\prime}+A=F_{B}^{\prime \prime}+A^{\prime} .
$$

Since $A \neq A^{\prime}$, we must have $A \in F_{B}^{\prime \prime}$. This implies $A<B$. Comparing $F=F_{A}+B$ to $G=F_{B}^{\prime}+A$ we see that every tree in $G$ is strictly less than $B$, which implies $F>G$, contrary to hypothesis. So this case is impossible.

Having shown that $G$ cannot exist, $\phi(T, F)$ has the desired properties.

Theorem 6.3. Let $T \geq T_{4}$ be given. Then the shuffle vectors in the set

$$
\{S(w): \operatorname{tree}(w)=T \text { and } w \text { is nondegenerate }\}
$$

are linearly independent.

Proof. Let $w_{1}<\cdots<w_{q}$ be the non-degenerate words which satisfy $\operatorname{tree}(w)=T$. We wish to prove that the vectors $S\left(w_{1}\right), \ldots, S\left(w_{q}\right)$ are linearly independent. Let $X_{1}<\cdots<$ $X_{p}$ be the list of trees with the same number of leaves as $T$. Let $A=\left(a_{i j}\right)$ be $p \times q$ matrix in which each column $j$ contains the coefficients of $S\left(w_{j}\right)$ expanded in terms of the ordered basis $X_{1}, \ldots, X_{p}$. Construct $G_{A}$ as in Definition 4.1. We define our row selection function

$$
r:\left\{w_{1}, \ldots, w_{q}\right\} \rightarrow\left\{X_{1}, \ldots, X_{p}\right\}
$$

via

$$
r\left(w_{i}\right)=\phi\left(T, F\left(w_{i}\right)\right)
$$

where $\phi\left(T, F\left(w_{i}\right)\right)$ is a tree which contains $F\left(w_{i}\right)$ and no larger forest of $T$, the existence of which is guaranteed by Proposition 6.2. We will show that $G_{r}$ has no non-trivial cycles, which implies $A$ has rank $q$ and that the shuffle vectors are linearly independent.

The non-loop edges in $G_{r}$ are of the form $\left(w_{i}, \phi\left(T, F\left(w_{i}\right)\right), w_{j}\right)$ where $w_{i}$ and $w_{j}$ are distinct words and $\phi\left(T, F\left(w_{i}\right)\right)$ appears in the support of both $S\left(w_{i}\right)$ and $S\left(w_{j}\right)$. This implies that $F\left(w_{i}\right)$ and $F\left(w_{j}\right)$ are both forests of $\phi\left(T, F\left(w_{i}\right)\right)$, and by 6.2 this implies $F\left(w_{i}\right) \geq F\left(w_{j}\right)$. Since $w_{i}$ and $w_{j}$ are distinct words generating $T$, they cannot be rearrangements of each other by Lemma 4.2 of [4]. Therefore $F\left(w_{i}\right)>F\left(w_{j}\right)$. Hence $F\left(v_{1}\right)>F\left(v_{2}\right)>\cdots$ along every walk along non-loop edges in $G_{r}$ from vertex $v_{1}$ to $v_{2}$ to ..., which implies that $G_{r}$ has no non-trivial cycles.

Corollary 6.4. Let $T$ be an arbitrary tree on $v$ vertices. Then the shuffle vectors in the set

$$
\left\{S(w): \operatorname{tree}(w)=T \text { and } w \neq t_{1}^{v-1}\right\}
$$

are linearly independent. 
Proof. Extend the vertex set of $G_{A}$ in the proof of Theorem 6.3 to include all instances of $w$ where $T=\operatorname{tree}(w)$ and $F(w)$ is degenerate and $w \neq t_{1}^{v-1}$. Extend the row selection function by defining $r(w)=T$ in each instance. Then $G_{r}$ cannot contain any non-loop edges of the form $\left(w_{i}, r\left(w_{i}\right), t_{a}^{j}\right)$ where $F\left(w_{i}\right)$ is non-degenerate because otherwise $r\left(w_{i}\right)$ is in the support of $S\left(t_{a}^{j}\right)=j ! T$, forcing $r\left(w_{i}\right)=T$, contradicting $\phi\left(T, F\left(w_{i}\right)\right) \neq T$. The only conceivable contribution to a non-trivial cycle in $G_{r}$ must therefore be of the form $\left(t_{a}^{j}, T, t_{b}^{k}\right)$, which implies that $T=\operatorname{tree}\left(t_{a}^{j}\right)=\operatorname{tree}\left(t_{b}^{k}\right)$ where $a \neq b$. This can only occur if $a=1$ or $b=1$, which is forbidden.

\section{Shuffles of Sufficient Length Forests}

The main result of this section is Theorem 7.15. Preliminaries:

Definition 7.1. A forest of trees $F$ has sufficient length if and only $F=F(w)$ for some word of length $d$, where tree $(w)$ has $\leq 2^{d}$ leaves.

Definition 7.2. The set of totally symmetric rooted binary trees is $\mathcal{S}=\left\{S_{0}, S_{1}, \ldots\right\}$, where $S_{0}=T_{1}$ and for $k \geq 1, S_{k}=\left\{S_{k-1}, S_{k-1}\right\}$. We denote by $s_{k}$ the variable $t_{n}$ such that $S_{k}=T_{n}$, hence $s_{0}=t_{1}, s_{1}=t_{2}, s_{2}=t_{4}, s_{3}=t_{26}$, etc.

Definition 7.3. Let $F$ be a forest of trees and let $k$ be a positive integer. Then $\operatorname{sym}_{k}(F)=$ $s_{e_{b}} \cdots s_{e_{1}}$ where $S_{e_{1}}+\cdots+S_{e_{b}}$ is the maximal subforest of $F$ such that $e_{i} \leq k-i-1$ for each $i$. If $F$ does not contain any totally symmetric trees of height $\leq k-2$ then we set $\operatorname{sym}_{k}(F)=1$.

Definition 7.4. Let $F$ be a forest of trees and let $k$ be a positive integer. The $k^{\text {th }}$ tree label for $F$ is

$$
\operatorname{treeLabel}_{k}(F)=\operatorname{tree}(\text { pmt }),
$$

where $p, m$, and $t$ are words in $t_{1}, t_{2}, \ldots$ defined as follows:

$$
\begin{gathered}
p= \begin{cases}1 & S_{0} \notin F \\
s_{0} \cdots s_{a-1} & a \text { is maximal such that } \sum_{i=0}^{a-1} S_{i} \subseteq F,\end{cases} \\
\quad t=\operatorname{sym}_{k}(F-F(p)),
\end{gathered}
$$

and

$$
m=\max \{w: F(w)=F-F(p)-F(t)\} .
$$

We will use the notation $p_{k}(F)=p, m_{k}(F)=m$, and $t_{k}(F)=t$. We will describe the subtrees of treeLabel ${ }_{k}(F)$ corresponding to $t_{k}(F)$ as tail trees and the subtrees of treeLabel $L_{k}(F)$ corresponding to $m_{k}(F)$ as middle trees. The symmetric consolidation of $F$ is tree $(p)$, which is equal to $s_{0}$ if $p=1$ and $s_{a}$ if $p=s_{0} \cdots s_{a-1}$. By construction of $p$, the symmetric consolidation of $F$ cannot belong to $F$. 
Example 7.5. Let $F=2 T_{1}+2 T_{2}+T_{3}+T_{26}=2 S_{0}+2 S_{1}+T_{3}+S_{3}$. Then

$$
\begin{gathered}
p_{3}(F)=s_{0} s_{1}, \\
t_{3}(F)=s_{0} s_{1}, \\
m_{3}(F)=s_{3} t_{3}, \\
\text { treeLabel }_{3}(F)=\operatorname{tree}\left(s_{0} s_{1} s_{3} t_{3} s_{0} s_{1}\right) .
\end{gathered}
$$

The symmetric consolidation of $F$ is tree $\left(s_{0} s_{1}\right)=s_{2}$. See Figure 7.1.

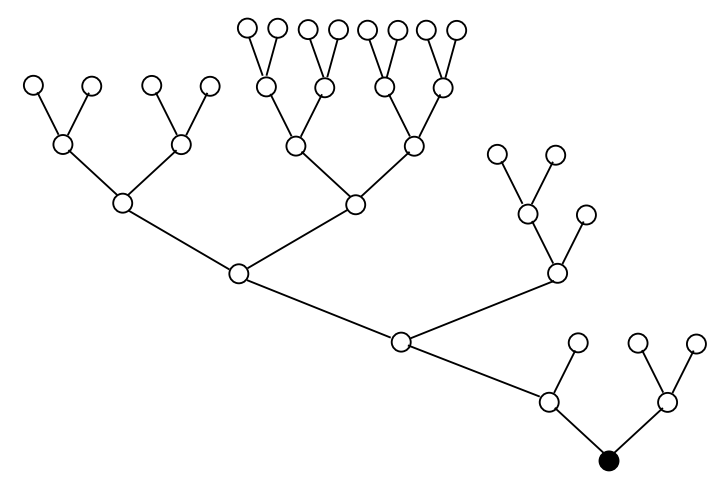

Figure 7.1: treeLabel $_{3}\left(2 T_{1}+2 T_{2}+T_{3}+T_{26}\right)$

Definition 7.6. Let $T$ be a rooted binary tree. The number of symmetry dots in $T$ is given by the statistic

$$
\operatorname{symDots}(T)= \begin{cases}1 & T=T_{1} \\ \operatorname{sym} \operatorname{Dots}(A)+\operatorname{symDots}(B)+\chi(T \in \mathcal{S}) & T=\{A, B\} .\end{cases}
$$

Given a tree $T$, the symDot statistic marks each vertex of $T$ which is the root of a totally symmetric subtree of $T$ with a dot, then counts the dots.

The symmetry dots of the tree in Figure 7.1 are depicted in red in Figure 7.2.

Definition 7.7. Let $F$ be a forest of trees and let $k$ be positive integer. We define the $k^{\text {th }}$ $Z$-statistic of $F$ to be triple

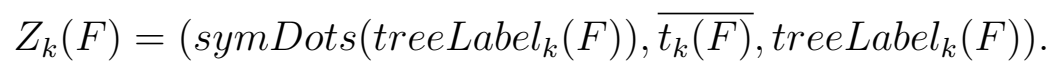

Since integers, words, and trees each have their own ordering, we can order such triples using lexicographic order. 


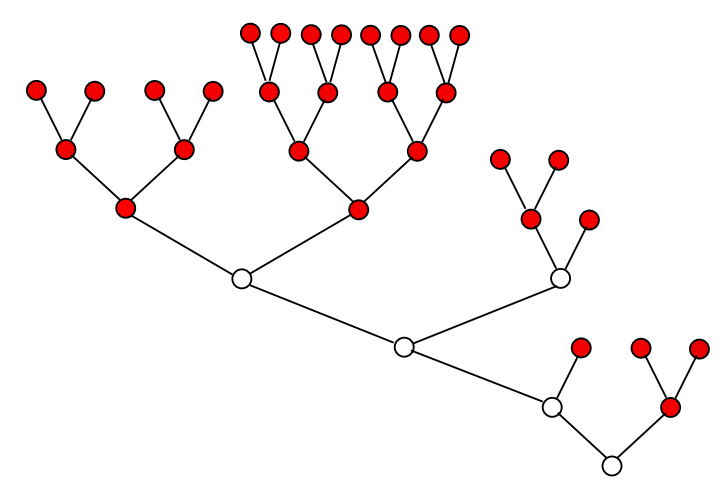

Figure 7.2: Symmetry Dots

Definition 7.8. Let $w=t_{e_{1}} \cdots t_{e_{k}}$ be a word in the variables $t_{1}, t_{2}, \ldots$ If $t_{e_{1}} \neq s_{0}$ then the symmetric prefix of $w$ is symFix $(w)=1$. But if $t_{e_{1}}=s_{0}$ then the symmetric prefix of $w$ is $\operatorname{symFix}(w)=s_{0} \cdots s_{a-1}$, where $a \geq 1$ is the maximum integer such that $t_{e_{i}}=s_{i-1}$ for $1 \leq i \leq a$.

Lemma 7.9. Let $u=t_{e_{1}} \cdots t_{e_{k}}$ be a word in $t_{1}, t_{2}, \ldots$ Then

$$
\operatorname{symDots}(\operatorname{tree}(u))=\sum_{i=1}^{n} \operatorname{symDots}\left(T_{e_{i}}\right)+\operatorname{deg}(\operatorname{symFix}(u))+1 .
$$

Proof. Let $T$ be an arbitrary rooted binary tree and let $x$ be an arbitrary leaf vertex of $T$. Let $x_{0}, \ldots, x_{r}$ be the vertices encountered in $T$ along the path from $x_{0}=x$ to the root vertex $x_{r}$. For $0 \leq i \leq r-1$ let $y_{i}$ be the sibling of $x_{i}$. For an arbitrary vertex $x$ in $T$ let $T(x)$ denote the subtree of $T$ rooted at $x$ and let $\sigma(x)=\chi(T(x) \in \mathcal{S})$. Then we have

$$
\operatorname{symDots}(T)=\sum_{i=0}^{r-1} \operatorname{symDots}\left(T\left(y_{i}\right)\right)+\sum_{i=0}^{r} \sigma\left(x_{i}\right) .
$$

If $\sigma\left(x_{a}\right)=1$ for some $a \geq 0$ then $\sigma\left(x_{b}\right)=1$ for $0 \leq b \leq a$, and if $\sigma\left(x_{a}\right)=0$ for some $a \leq r$ then $\sigma\left(x_{b}\right)=0$ for all $a \leq b \leq r$. We always have $\sigma\left(x_{0}\right)=1$.

Now consider $T=\operatorname{tree}(u)$. Then there is a leaf vertex $x$ of $T$ such that if $x_{0}, \ldots, x_{r}$ are the vertices encountered in $T$ along the path from $x_{0}=x$ to the root vertex $x_{r}$ and if $y_{i}$ is the sibling of $x_{i}$ for each $i \leq r-1$, then we have $T\left(y_{i}\right)=T_{e_{i}}$ for each $i$. The observations in the previous paragraph imply that

$$
\sum_{i=0}^{r} \sigma\left(x_{i}\right)=1+\operatorname{deg}(\operatorname{symFix}(u)),
$$

and the formula for symDots(tree $(u))$ is a consequence of this. 
Lemma 7.10. Let $F$ be a forest of trees and assume $s_{a}$ appears in $m_{k}(F)$ for some $a \leq k-2$. Then $s_{a}$ also appears in $t_{k}(F)$.

Proof. The word $t_{k}(F)$ is constructed by first deleting from $F$ the largest run of totally symmetric trees, then forming the largest subforest of the form $S_{e_{1}}+\cdots+S_{e_{b}}$ from the remaining subforest $G$, where $e_{i} \leq k-i-1$ for each $i$. Suppose $s_{a}$ does not appear in $t_{k}(F)$. Then either $k-a-1>b$ or $S_{e_{k-a-1}}<S_{a}$. Since $s_{a}$ appears in $m_{k}(F), S_{a}$ must appear in $G$. But $S_{e_{1}}+\cdots+S_{e_{b}}$ can be made larger by adding $S_{a}$ to it (if $k-a-1>b$ ) or replacing $S_{e_{k-a-1}}$ by $S_{a}$, and this contradicts the construction of $t_{k}(F)$. Therefore in fact $s_{a}$ must appear in $t_{k}(F)$.

Lemma 7.11. Let $F<G$ be forests of sufficient length, each appearing in trees on $v$ leaves where $2^{k-1}+1 \leq v \leq 2^{k}$. Then $\operatorname{treeLabel}_{k}(F) \neq$ treeLabel $_{k}(G)$.

Proof. Suppose in fact that $\operatorname{treeLabel}_{k}(F)=\operatorname{treeLabel}_{k}(G)=T$. Then we can identify two distinct leaf vertices $x$ and $y$ in $T$ that give rise to $F$ and $G$, respectively. Each vertex defines a path to the root vertex of $T$. Suppose these paths intersect in the vertex $z$. Let the subtree of $T$ rooted at $z$ be $Z=\{X, Y\}$ and assume that $x$ appears as a leaf of $X$ and that $y$ appears as a leaf in $Y$. See Figure 7.3. We can now say that $F=F_{X}+Y+H$ and $G=F_{Y}+X+H$, where $F_{X}$ is the forest of $X$ defined by $x, F_{Y}$ is the forest of $Y$ defined by $Y$, and $H=F-F_{X}-Y=G-F_{Y}-X$. The assumption that $F<G$ implies that $X>Y$.

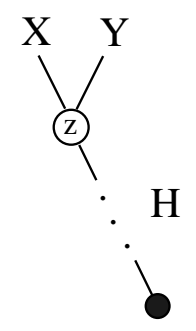

Figure 7.3: Path Intersection in Lemma 7.11

The tails $t_{k}(F)$ and $t_{k}(G)$ are constructed in such a way that all leaf vertices of subtrees of $T$ corresponding to them have height $\leq k-1$ as vertices of $T$. Since $F$ and $G$ are forests of sufficient length in a tree with at least $2^{k-1}+1$ vertices, both $x$ and $y$ are leaves of height $\geq k$ in $T$. Hence $X$ is a middle tree of $G$ and $Y$ is a middle tree of $F$ and all tail trees of $F$ and $G$ fall in $H$. We claim as a consequence of this that $Y$ is a totally symmetric tree. If $Y$ is not totally symmetric then it is not the symmetric consolidation of $G$. If we therefore write $Y=\left\{Y_{1}, Y_{2}\right\}$, where $y$ appears in $Y_{1}$, then by construction of $\operatorname{treeLabel}_{k}(G)$ we must have $X \leq Y_{2}$ since both are middle trees of $G$. This contradicts $X>Y$. Therefore $Y$ must be totally symmetric and the consolidation of the symmetric run in $G$ is $Y$. This implies that $Y \notin H$, therefore $Y \geq S_{k-1}$ as a middle tree of $F$ by Lemma 7.10. Since $X>Y$ and $v \leq 2^{k}$, this is impossible. Therefore $\operatorname{treeLabel}_{k}(F) \neq \operatorname{treeLabel}_{k}(G)$. 
Lemma 7.12. Let $x, y$, and $z$ be words in $t_{1}, t_{2}, \ldots$ Assume tree $(x)<\operatorname{tree}(y)$. Then $\operatorname{tree}(x z)<\operatorname{tree}(y z)$.

Proof. By induction on the length of $z$. The base case, length zero, is trivial. Assume the lemma is true for length $k$. Let $z$ be a word of length $k+1$, and write $z=z_{0} t_{i}$. By the induction hypothesis, $\operatorname{tree}\left(x z_{0}\right)<\operatorname{tree}\left(y z_{0}\right)$, therefore

$$
\operatorname{tree}(x z)=\left\{\operatorname{tree}\left(x z_{0}\right), T_{i}\right\}<\left\{\operatorname{tree}\left(y z_{0}\right), T_{i}\right\}=\operatorname{tree}(y z)
$$

Lemma 7.13. Let $w$ be a non-trivial word in $t_{1}, t_{2}, \ldots$ Let $u$ and $v$ be words in $t_{1}, t_{2}, \ldots$ Then tree $(u w v) \leq \operatorname{tree}(u \bar{w} v)$.

Proof. By Lemma 7.12 it suffices to prove this for $v=1$. We will proceed by induction on the length of $w$. The statement is true for length 1 . Now consider length $k \geq 2$. Write $w=t_{e_{1}} t_{e_{2}} \cdots t_{e_{k}}$. Let $a$ be the maximal index such that $e_{a}$ is minimal in $\left\{e_{1}, e_{2}, \ldots, e_{k}\right\}$. There are two cases to consider: $a=k$ and $a<k$.

Case 1. If $a=k$, write $w=w_{0} t_{e_{k}}$. By the induction hypothesis $\operatorname{tree}\left(u w_{0}\right) \leq \operatorname{tree}\left(u \overline{w_{0}}\right)$, therefore by Lemma 7.12 we have

$$
\operatorname{tree}(u w)=\operatorname{tree}\left(u w_{0} t_{e_{k}}\right) \leq \operatorname{tree}\left(u \overline{w_{0}} t_{e_{k}}\right)=\operatorname{tree}(u \bar{w})
$$

Case 2. If $a<k$ then $e_{a}<e_{k}$. Write $w=x t_{e_{a}} y t_{e_{k}}$. We have

$$
\operatorname{tree}\left(u x t_{e_{a}}\right)=\left\{\operatorname{tree}(u x), T_{e_{a}}\right\}<\left\{\operatorname{tree}(u x), T_{e_{k}}\right\}=\operatorname{tree}\left(u x t_{e_{k}}\right),
$$

hence by Lemma 7.12

$$
\operatorname{tree}\left(u x t_{e_{a}} y\right)<\operatorname{tree}\left(u x t_{e_{k}} y\right)
$$

We also have

$$
T_{e_{k}}<\operatorname{tree}\left(u x t_{e_{k}} y\right)
$$

Therefore

$$
\operatorname{tree}(u w)=\left\{\operatorname{tree}\left(u x t_{e_{a}} y\right), T_{e_{k}}\right\}<\left\{\operatorname{tree}\left(u x t_{e_{k}} y\right), T_{e_{a}}\right\} .
$$

By the induction hypothesis,

$$
\operatorname{tree}\left(u x t_{e_{k}} y\right) \leq \operatorname{tree}\left(u \overline{x t_{e_{k}} y}\right) .
$$

Therefore

$$
\left\{\operatorname{tree}\left(u x t_{e_{k}} y\right), T_{e_{a}}\right\} \leq\left\{\operatorname{tree}\left(u \overline{x t_{e_{k}} y}\right), T_{e_{a}}\right\}=\operatorname{tree}\left(u \overline{x t_{e_{k}} y} t_{e_{a}}\right)=\operatorname{tree}(u \bar{w}) .
$$

Hence $\operatorname{tree}(u w)<\operatorname{tree}(u \bar{w})$. 
Proposition 7.14. Let $F$ and $G$ be distinct forests in trees with $v$ leaves, $2^{k-1}+1 \leq v \leq$ $2^{k}$. If $G$ is a forest of treeLabel $(F)$ then $Z_{k}(G)>Z_{k}(F)$.

Proof. Write

$$
\begin{gathered}
T=\operatorname{treeLabel}_{k}(F)=\operatorname{tree}(p m t), \\
T^{\prime}=\operatorname{treeLabel}_{k}(G)=\operatorname{tree}\left(p^{\prime} m^{\prime} t^{\prime}\right),
\end{gathered}
$$

where $p=p_{k}(F), m=m_{k}(F), t=t_{k}(F), p^{\prime}=p_{k}(G), m^{\prime}=m_{k}(G)$, and $t^{\prime}=t_{k}(G)$ are constructed as in Definition 7.4. Let $w=p m t$. Since $G$ is a forest of $T$, there must exist a rearrangement $w^{\prime}$ of $p^{\prime} m^{\prime} t^{\prime}$ such that $T=\operatorname{tree}\left(w^{\prime}\right)$.

By the tree label construction, symFix $\left(p^{\prime} m^{\prime} t^{\prime}\right)=p^{\prime} \geq \operatorname{symFix}\left(w^{\prime}\right)$, and by Lemma 7.9 this implies $\operatorname{symDots}\left(T^{\prime}\right) \geq \operatorname{symDots}(T)$. Moreover $p^{\prime}>\operatorname{symFix}\left(w^{\prime}\right)$ implies symDots $\left(T^{\prime}\right)>$ $\operatorname{symDots}(T)$, which in turn implies $Z_{k}(G)>Z_{k}(F)$. So we can assume without loss of generality that $p^{\prime}=\operatorname{symFix}\left(w^{\prime}\right)$ and $\operatorname{symDots}\left(T^{\prime}\right)=\operatorname{symDots}(T)$.

Write $m=t_{e_{c}} \cdots t_{e_{1}}$ where $e_{c} \geq \cdots \geq e_{1}$. By construction of $T$, every leaf vertex in subtrees of $T$ corresponding to $t_{k}(F)$ has height $\leq k-1$ in $T$. Since $\operatorname{tree}(p)$ is a totally symmetric tree, every leaf vertex of $T$ originating in the subtree $\operatorname{tree}(p)$ gives rise to the forest $F$. Hence $G$ must be generated by a leaf vertex $y$ corresponding to the subtree $T_{e_{i}}$ of $T$, one of the middle trees of $F$. This implies that if $x$ is the leaf vertex in $T$ that gives rise to $F$, then the paths from $x$ and $y$ to the root of $T$ intersect in the parent, $z$, of the root of $T_{e_{i}}$. See Figure 7.4. Therefore $F(t)$, the tail trees in the treelabel for $F$, is a subforest of $G$.

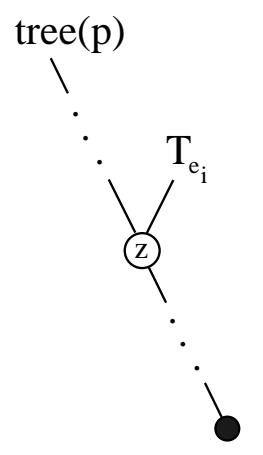

Figure 7.4: Path Intersection in Proposition 7.14

We claim that $F(t)$ is a subforest of $G-F\left(p^{\prime}\right)$. The reason for this is that there cannot be a symmetry dot at $z$ for one of two reasons: If $i<c$ then the subtree of $T$ rooted at the sibling of the root of $T_{e_{i}}$ contains $T_{e_{c}} \geq T_{e_{i}}$ as a proper subtree, and if $i=c$ then there cannot be a symmetry dot at $z$ because $T_{e_{c}} \neq \operatorname{tree}(p)$ (otherwise tree $(p)$ cannot be the symmetric consolidation of $F)$. Hence $F(t) \subseteq G-F\left(p^{\prime}\right)$. This implies that $\bar{t}^{\prime} \geq \bar{t}$ by construction of $t^{\prime}=t_{k}(G)$. If $\overline{t^{\prime}}>\bar{t}$ then combined with $\operatorname{symDots}\left(T^{\prime}\right)=\operatorname{symDots}(T)$ 
we have $Z_{k}(G)>Z_{k}(F)$. So we can assume without loss of generality that both $p^{\prime}=$ $\operatorname{symFix}\left(w^{\prime}\right)$ and $\overline{t^{\prime}}=\bar{t}$. The latter equality implies $t^{\prime}=t$.

We can now now write $w^{\prime}=p^{\prime} \sigma\left(m^{\prime}\right) t^{\prime}$ where $\sigma\left(m^{\prime}\right)$ is a rearrangement of $m^{\prime}$ and $m^{\prime}=\overline{\sigma\left(m^{\prime}\right)}$. Therefore

$$
T^{\prime}=\operatorname{tree}\left(p^{\prime} m^{\prime} t^{\prime}\right) \geq \operatorname{tree}\left(p^{\prime} \sigma\left(m^{\prime}\right) t^{\prime}\right)=T
$$

by Lemma 7.13. Since $T^{\prime} \neq T$ by Lemma 7.11, we must in fact have $T^{\prime}>T$. Combined with $\operatorname{sym} \operatorname{Dots}\left(T^{\prime}\right)=\operatorname{symDots}(T)$ and $t^{\prime}=t$ this implies $Z_{k}(G)>Z_{k}(F)$.

Theorem 7.15. The shuffle vectors in the set

$$
\{S(w): F(w) \text { is a forest of sufficient length }\}
$$

are linearly independent.

Proof. Fix $k \geq 1$ and $2^{k-1}+1 \leq v \leq 2^{k}$. It will suffice to restrict our attention to shuffle vectors of trees with $v$ leaves. Let $w_{1}<\cdots<w_{q}$ be the list of words in $t_{1}, t_{2}, \ldots$ which generate the distinct shuffle vectors of sufficient length on $v$ leaves. We wish to prove that the vectors $S\left(w_{1}\right), \ldots, S\left(w_{q}\right)$ are linearly independent. Let $X_{1}<\cdots<X_{p}$ be the list of trees with $v$ leaves. Let $A=\left(a_{i j}\right)$ be $p \times q$ matrix in which each column $j$ contains the coefficients of $S\left(w_{j}\right)$ expanded in terms of the ordered basis $X_{1}, \ldots, X_{p}$. Construct $G_{A}$ as in Definition 4.1. We define our row selection function

$$
r:\left\{w_{1}, \ldots, w_{q}\right\} \rightarrow\left\{X_{1}, \ldots, X_{p}\right\}
$$

via

$$
r\left(w_{i}\right)=\operatorname{treeLabel}_{k}\left(F\left(w_{i}\right)\right)
$$

where $\operatorname{tree~} \operatorname{Label}_{k}\left(F\left(w_{i}\right)\right)$ is the tree having $F\left(w_{i}\right)$ as one its forests as constructed in Definition 7.4. We will show that $G_{r}$ has no non-trivial cycles, which implies $A$ has rank $q$ and that the shuffle vectors are linearly independent.

The non-loop edges in $G_{r}$ are of the form

$$
\left(S\left(w_{i}\right), T, S\left(w_{j}\right)\right)
$$

where $F\left(w_{i}\right)$ and $F\left(w_{j}\right)$ are distinct forests of sufficient length and

$$
T=\text { treeLabel }_{k}\left(F\left(w_{i}\right)\right)
$$

appears in the support of both $S\left(w_{i}\right)$ and $S\left(w_{j}\right)$. This implies that $F\left(w_{i}\right)$ and $F\left(w_{j}\right)$ are both forests appearing in $T$. By Proposition 7.14, we must have $Z_{k}\left(F\left(w_{i}\right)\right)<Z_{k}\left(F\left(w_{j}\right)\right)$. Hence the $Z_{k}$-statistic of the multisets represented by the vertices strictly increases along every walk using non-loop edges in $G_{r}$, which implies that $G_{r}$ has no non-trivial cycles. 


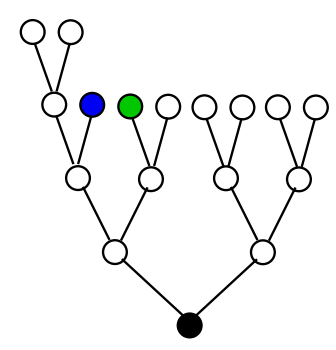

Figure 8.1: Insufficient Length Forests, Same Tree Label

\section{Sufficient Length is Necessary}

We have used the acyclic digraph method to prove that our coefficient matrices in Theorems 5.3, 6.3, and 7.15 have full rank. Essentially what we are doing is arguing that one can find a square submatrix of full rank which is permutationally equivalent to an upper-triangular matrix with non-zero entries along the diagonal. This method is sufficient for proving full rank, but not necessary, because there are matrices which do not meet this condition that have full rank. But the method is necessary if we do not wish to sully our hands with the actual coefficients and instead just deal with the distribution of zero and non-zero entries. Sufficient length is used in Theorem 7.15 to guarantee that tree Label $_{k}$ is injective on forests of length $\geq k$ when the forests appear in trees with $v$ vertices, $2^{k-1}+1 \leq v \leq 2^{k}$, and this is necessary to avoid cycles of length 2 in $G_{r}$. There are an infinite pair of forests $F<G$ of length $k-1$ which violate this condition when $k \geq 3:$

Example 8.1. Let $T_{a}$ be a tree with $v^{\prime}$ vertices, $2 \leq v^{\prime} \leq 2^{k-1}+1$. Let

$$
\begin{gathered}
T=\operatorname{tree}\left(t_{a} s_{1} \cdots s_{k-2}\right), \\
F=S_{1}+\cdots+S_{k-2}+T_{a}, \\
G=S_{0}+S_{2}+\cdots+S_{k-2}+\operatorname{tree}\left(t_{a}\right) .
\end{gathered}
$$

Then $T$ has $v$ leaves, $2^{k-1}+1 \leq v \leq 2^{k}$, and

$$
\operatorname{treeLabel}_{k}(F)=T=\operatorname{treeLabel}_{k}(G) \text {. }
$$

In Figure 8.1 we illustrate this construction for $k=4, T_{a}=T_{2}=S_{1}$. The forests $F=2 S_{1}+S_{2}$ and $G=S_{0}+T_{3}+S_{2}$ have the same treelabel. $F$ originates from the blue vertex and $G$ originates from the green vertex. The treelabel has 9 vertices, hence neither $F$ nor $G$ has sufficient length. 


\section{References}

[1] H. Bass, E. H. Connell and D. Wright, The Jacobian conjecture: reduction of degree and formal expansion of the inverse, Bull. Amer. Math. Soc. 7 (2) (1982), 287-330.

[2] A. Joyal, Une théorie combinatoire des séries formelles, Adv. in Math. 42, No. 1, 1981.

[3] O.H. Keller, Ganze Cremona-Transformationen, Monatsh. Math. Phys., 47(1939), 229-306.

[4] D. Singer, On Catalan trees and the Jacobian conjecture, The Electronic Journal of Combinatorics 8(1) (2001), \#R2.

[5] D. Singer, A graph-theoretic method for choosing a spanning set for a finitedimensional vector space, with applications to the Grossman-Larson-Wright module and the Jacobian conjecture, The Electronic Journal of Combinatorics 16(1) (2009), \#R43.

[6] S. Wang, A Jacobian criterion for separability, J. Algebra 65 (1980), 453-494.

[7] D. Wright, Reversion, trees, and the Jacobian conjecture, Combinatorial and computational algebra (Hong Kong, 1999), 249-267, Contemp. Math. 264, Amer. Math. Soc., Providence, RI, 2000.

[8] D. Wright, The Jacobian conjecture as a problem in combinatorics, arXiv:math.CO/0511214v2 22 Mar 2006.

[9] D. Zeilberger, Toward a combinatorial proof of the Jacobian conjecture?, Proc. of the "Colloque de combinatoire enumerative," G.Labelle and P.Leroux, editors, Lecture Notes in Math. 1234, 370-380, Springer-Verlag, Berlin (1987). 